\title{
MATHEMATICAL MODELING FOR THE EXTRACTION OF URANIUM AND MOLYBDENUM WITH EMULSION LIQUID MEMBRANE, INCLUDING INDUSTRIAL APPLICATION AND COST EVALUATION OF THE URANIUM RECOVERY
}

\author{
KRIS TRI BASUKI \\ Sekolah Tinggi Teknologi Nuklir \\ Badan Tenaga Nuklir Nasional - BATAN \\ JL.Babarsari Kotak Pos 1008, Yogyakarta 55010
}

\begin{abstract}
MATHEMATICAL MODELING FOR THE EXTRACTION OF URANIUM AND MOLYBDENUM WITH EMULSION LIQUID MEMBRANE, INCLUDING INDUSTRIAL APPLICATION AND COST EVALUATION OF THE URANIUM RECOVERY. Emulsion liquid membrane systems are double emulsion drops. Two immiscible phases are separated by a third phase which is immiscible with the other two phases. The liquid membrane systems were classified into two types: (1) carrier mediated mass transfer, (2) mass transfer without any reaction involved. Uranium extraction, molybdenum extraction and solvent extraction were used as purposed elements for each type of the membrane systems in the derivation of their mathematical models. Mass transfer in emulsion liquid membrane (ELM) systems has been modeled by several differential and algebraic equations. The models take into account the following : mass transfer of the solute from the bulk external phase to the external phase-membrane interface; an equilibrium reaction between the solute and the carrier to form the solutecarrier complex at the interface; mass transfer by diffusion of the solute-carrier complex in the membrane phase to the membrane-internal phase interface; another equilibrium reaction of the solute-carrier complex to release the solute at the membrane-internal phase interface into the internal phase. Models with or without the consideration of film resistances were developed and compared. The models developed in this study can predict the extraction rate through emulsion liquid membranes theoretically. All parameters required in the models can be determined before an experimental extraction run. Experimental data from literature (uranium extraction) and (molybdenum extraction and solvent extraction) were used to test the models. The agreements between the theoretical predictions and the experimental data were very good. The advantages of emulsion liquid membrane systems over traditional methods were discussed. The models developed in this research can be used directly for the design of emulsion liquid membrane systems. The results of this study represent a very significant step toward the practical applications of the emulsion liquid membrane technology.
\end{abstract}

Keywords : emulsion liquid membrane, uranium, molybdenum, mathematical modeling, $\mathrm{D}_{2}$ EHPA, mass transfer, span 80 , batch process, recovery, cost 


\begin{abstract}
Abstrak
PEMODELAN MATEMATIKA UNTUK EKSTRAKSI URANIUM DAN MOLIBDENUM DENGAN MEMBRAN EMULSI CAIR, YANG MENCAKUP APLIKASI INDUSTRI DAN EVALUASI BIAYA PEMUNGUTAN KEMBALI URANIUM. Sistem membran emulsi cair merupakan tetesan emulsi ganda. Dua fase tak pisah dipisahkan oleh fase ketiga yang tak pisah dengan dua fase lainnya. Sistem-sistem membran cair dikelompokkan dalam dua jenis : (1) perpindahan massa bermedium pengangkut, (2) perpindahan panas tanpa ada reaksi apapun yang terlibat. Ekstraksi uranium, ekstraksi molibdenum dan ekstraksi pelarut digunakan sebagai element bermanfaat untuk setiap jenis sistem membran dalam turunan model matematikanya. Perpindahan massa dalam sistem membran emulsi cair (ELM) telah dimodelkan dengan beberapa persamaan diferensial dan aljabar. Model-model ini menggunakan ketentuan berikut : perpindahan massa zat terlarut dari fase luar yang berukuran besar ke antarmuka fase membran luar; suatu reaksi keseimbangan antara zat terlarut dan pengangkut untuk membentuk kompleks zat-terlarut-dan-pengangkut pada antarmuka; perpindahan massa dengan difusi dari kompleks zat-terlarut-dan-pengangkut dalam fase membran ke antarmuka fase membran internal; reaksi keseimbangan lain dari kompleks zat-terlarutdan-pengangkut untuk melepaskan zat terlarut pada antarmuka fase membran internal ke fase internal. Model dengan atau tanpa pertimbangan resistansi film telah dikembangkan dan dibandingkan. Model yang dikembangkan dalam penelitian ini secara teori dapat memprediksi rerata ekstraksi melalui membran emulsi cair. Semua parameter yang diperlukan dalam model ini dapat ditetapkan sebelum sebuah ekstraksi percobaan dijalankan. Data percobaan dari literatur (ekstraksi uranium, ekstraksi molibdenum dan ekstraksi pelarut) digunakan untuk menguji model-model. Kesesuaian antara tebakan teoritis dan data percobaan sangat bagus. Keuntungan sistem membran emulsi cair ini atas metode tradisional didiskusikan dalam pembahasan. Model-model yang dikembangkan dalam penelitian ini menunjukkan langkah yang sangat signifikan menuju aplikasi praktis dari teknologi membran emulsi cair.
\end{abstract}

Keywords : Membran emulsi cair, uranium, molibdenum, pemodelan matematis, $\mathrm{D}_{2}$ EHPA, perpindahan massa, span 80 , batch process, biaya recovery

\title{
INTRODUCTION
}

The field of liquid membrane technology is currently undergoing a rapid expansion of the areas of both research and industrial separation techniques $^{[3,7,14,20,21,23,24]}$. Emulsion liquid membranes can be manipulated to selectively separate a specific solute from a mixture, and even to extract a solute against its concentration gradient ${ }^{[1,2,4,10]}$. Emulsion liquid membrane systems are comprised of three liquid phases. Two of these are miscible with each other but separated by a third phase (the membrane phase) which is immiscible with both. Mass is transferred from one of the miscible phase across the liquid membrane to the second miscible phase. In general, liquid membranes are either supported or unsupported. Supported liquid membranes can be held in a porous structure or bounded on either side by two thin polymeric films. Emulsion (unsupported) liquid membranes are usually in the form of double emulsion drops. For a water/oil/water (W/O/W) system, it is the immiscible oil phase, separating the two aqueous phases. For an $\mathrm{O} / \mathrm{W} / \mathrm{O}$ system, the liquid membrane is the 
immiscible water phase which separates the two oil phases. The effectiveness of the emulsion liquid membrane process can be enhanced by utilizing a facilited transport mechanism to maximize both the flux through the membrane phase, and the capacity of the receiving phase for the diffusing species ${ }^{[6,8,9,11,22]}$.

The aim of this paper is to study mass transfer modeling of uranium and molybdenum extraction though emulsion liquid membrane systems (ELM), where several differential and algebraic equations were derived considering the conditions of operation for the extraction of both uranium and molybdenum, for which the data were adapted from several literatures.

The emulsion liquid membrane process is unique and different from other membrane processes such as reverse osmosis or ultrafiltration. The membrane is a liquid phase involving an emulsion configuration. Emulsion liquid membranes (ELMs), also called surfactant liquid membranes are essentially double emulsions, i.e., water/oil/water $(\mathrm{W} / \mathrm{O} / \mathrm{W})$ systems or oil/water/oil $(\mathrm{O} / \mathrm{W} / \mathrm{O})$ systems. For the $\mathrm{W} / \mathrm{O} / \mathrm{W}$ systems, the oil phase separating the two aqueous phases is the liquid membrane. For the $\mathrm{O} / \mathrm{W} / \mathrm{O}$ systems, the liquid membrane is the water phase that is between the two oil phases. Since their discovery over two decades ago, emulsion liquid membranes have demonstrated considerable potential as effective tools for a wide variety of separations ${ }^{[12,13,16,17,18]}$. Two commercial applications are: the removal of zinc from wastewater in the viscose fiber industry and the removal of phenol from wastewater. Another application has been as a well control fluid for preventing well blowout and sealing loss zones in oil and gas wells. In addition to other proposed oil-field applications, ELMs also have potential utility as membrane reactors incorporating simultaneous separation and reaction processes. This utility includes the use of ELMs for controlling chemical reactions. The effectiveness of ELMs is a result of two facilitated mechanisms: type 1, in which the reaction in the receiving phase (the internal phase if the external phase is a feed) maintains a solute concentration of effectively zero; and type 2 (carrier facilitated transport), where the diffusing species is carried across the membrane phase by incorporating a 'carrier' compound (complexing agent or extractant) in the membrane phase. For both types of facilitated mechanisms, simultaneous extraction and stripping take place in a single step rather than two steps as in solvent extraction. As shown in following Figure (1), here two immiscible phases, water and oil for example, are mixed vigorously and emulsion droplets are formed (droplet size about 0.5-10 $\mu \mathrm{m})$, which are stabilized by the addition of a surfactant ${ }^{[1,4,15]}$. 


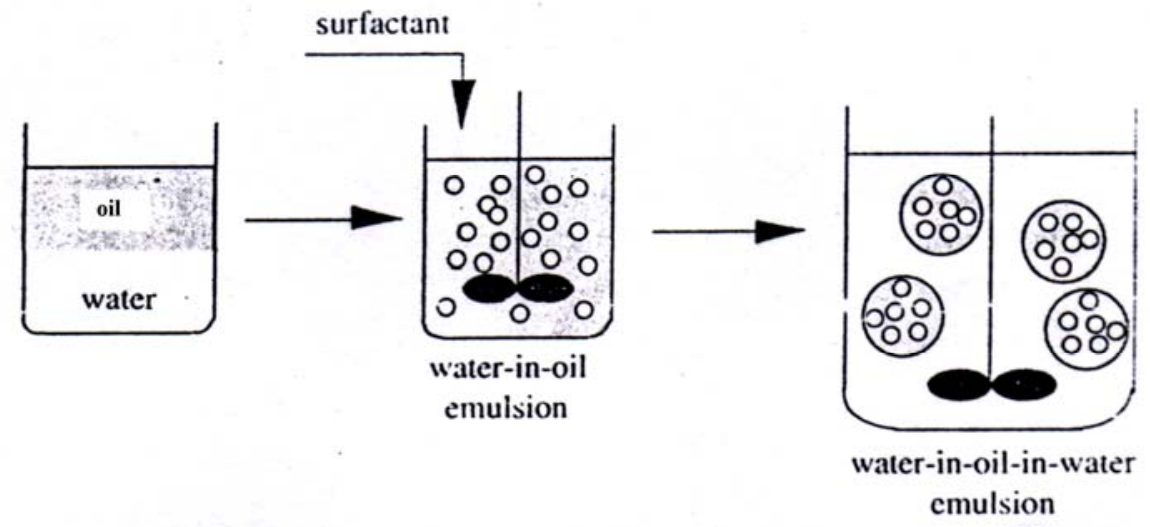

Fig. 1. Preparation of Emulsion Type Liquid Membrane (ELM)

\section{EXPERIMENT METHOD}

In this research $\mathrm{D}_{2}$ EHPA (di-2-ethylhexyl phosphoric acid) is used as carrier to mediate the uranium and molybdenum extraction. The membrane phase consists of $2 \% \mathrm{~W} \mathrm{D}_{2}$ EHPA (abbreviated as $(\mathrm{RH})_{2}$ ), $5 \% \mathrm{~V}$ surfactant Span 80 and the rest is kerosene. $\mathrm{D}_{2} \mathrm{EHPA}$ is a water insoluble liquid ion exchange material, one which forms a strong and selective complex with uranium and molybdenum. The uranium and molybdenum ions are insoluble in the membrane phase but the complexes are soluble in the membrane phase. These complexes diffuse through the membrane and react with the strong acid in the internal receiving phase. The uranium and molybdenum ions are trapped and concentrated in the receiving phase. These concentrated uranium and molybdenum ion solution are then easier for recovery or disposal. After the reaction of the complexes and the strong acid, the carriers are reformed and diffuse back to the membrane external interphase. By properly controlling the $\mathrm{pH}$ values on both sides of the membrane, uranium can be extracted from the low concentration external phase to the high concentration internal phase.

Models with or without the consideration of film resistances were developed and compared. The models developed in this study can predict the extraction rate through emulsion liquid membranes theoretically. All parameters required in the models can be determined before an experimental extraction run. Experimental data from literature (uranium extraction) and (molybdenum extraction and solvent extraction) were used to test the models.

An overall extraction equilibrium formulations for uranium/molybdenum and $\mathrm{D}_{2}$ EHPA are expressed as follows:

$$
\mathrm{UO}_{2}^{2+}+2(\mathrm{RH})_{2} \longleftrightarrow\left[\mathrm{UO}_{2} \mathrm{R}_{2} 2 \mathrm{HR}\right]+2 \mathrm{H}^{+}
$$




$$
\mathrm{MoO}_{2}^{2+}+2(\mathrm{RH})_{2} \longleftrightarrow\left[\mathrm{MoO}_{2} \mathrm{R}_{2} 2 \mathrm{HR}\right]+2 \mathrm{H}^{+}
$$

Equilibrium constants can be expressed as follows:

$$
\begin{aligned}
\mathrm{K}_{\mathrm{eq}} & =\frac{\left[\overline{\mathrm{MoO}_{2} \mathrm{R}_{2} 2 \mathrm{HR}}\right]\left[\mathrm{H}^{+}\right]^{2}}{\left[\mathrm{MoO}_{2}^{2+}\right]\left[\overline{\mathrm{RH})_{2}}\right]^{2}} \\
\mathrm{~K}_{\mathrm{eq}} & =\frac{\left[\overline{\mathrm{UO}_{2} \mathrm{R}_{2} 2 \mathrm{HR}}\right]\left[\mathrm{H}^{+}\right]^{2}}{\left[\mathrm{UO}_{2}^{2+}\right]\left[\overline{\mathrm{RH})_{2}}\right]^{2}}
\end{aligned}
$$

\section{Both the Membrane External and Internal Film Mass Transfer Resistances} are Neglected

In this case both the membrane external interphase mass transfer resistance and the membrane internal interphase mass transfer resistance are neglected. From the principal of material balance the governing equations that describe the concentrations of uranium, carrier and complex in each phases are ${ }^{[3]}$ :

Membrane phase:

$$
(1-\varepsilon) \frac{\partial \mathrm{C}_{\mathrm{X}}}{\partial \mathrm{t}}=\mathrm{D}_{\mathrm{eX}}\left(\frac{1}{\mathrm{r}^{2}} \frac{\partial}{\partial \mathrm{r}}\left(\mathrm{r}^{2} \frac{\mathrm{C}_{\mathrm{X}}}{\mathrm{r}}\right)\right)-\mathrm{R}_{\mathrm{X}}
$$

$\mathrm{t}=0 \quad \mathrm{C}_{\mathrm{X}}=0$ for all $\mathrm{r}$

$\mathrm{r}=0 \quad \mathrm{C}_{\mathrm{X}}=$ finite

$\mathrm{r}=\mathrm{R} \quad \mathrm{C}_{\mathrm{X}}=\mathrm{C}_{\mathrm{X}}^{*}$

$$
(1-\varepsilon) \frac{\partial \mathrm{C}_{(\mathrm{RH})_{2}}}{\partial \mathrm{t}}=\mathrm{D}_{\mathrm{e}(\mathrm{RH})_{2}}\left(\frac{1}{\mathrm{r}^{2}} \frac{\partial}{\partial \mathrm{r}}\left(\mathrm{r}^{2} \frac{\partial \mathrm{C}_{(\mathrm{RH})_{2}}}{\partial \mathrm{r}}\right)\right)-2 \mathrm{R}_{\mathrm{X}}
$$

$\mathrm{t}=0 \quad \mathrm{C}_{(\mathrm{RH}) 2}=\mathrm{C}_{(\mathrm{RH}) 2 \mathrm{O}}$ for all $\mathrm{r}$

$\mathrm{r}=0 \quad \mathrm{C}_{(\mathrm{RH}) 2}=$ finite

$\mathrm{r}=\mathrm{R} \quad \mathrm{C}_{(\mathrm{RH}) 2}=\mathrm{C}_{(\mathrm{RH}) 2}{ }^{*}$

External source phase:

$$
\mathrm{V}_{\mathrm{t}}\left(1-\phi^{\prime}\right) \frac{\mathrm{dCu}}{\mathrm{dt}}=\mathrm{N}\left(4 \pi \mathrm{R}^{2}\right) \mathrm{D}_{\mathrm{ex}}\left(\frac{\partial \mathrm{C}_{\mathrm{X}}}{\partial \mathrm{r}}\right)_{\mathrm{r}=\mathrm{R}}
$$

$\mathrm{t}=0 \quad \mathrm{Cu}=\mathrm{Cuo}$

Internal receiving phase:

$$
\varepsilon \frac{\partial \mathrm{Cui}}{\partial \mathrm{t}}=\mathrm{R}_{\mathrm{X}}
$$


$\mathrm{t}=0 \quad \mathrm{Cui}=0$

After solving the above equations; considering the boundary conditions, the following mathematical models were obtained:

$$
\begin{aligned}
& \frac{\mathrm{Cu}}{\mathrm{Cuo}}=\frac{3}{3+\mathrm{m} \Phi \mathrm{b}}+\sum_{n=1}^{\infty} \frac{\tan \left(\beta_{\mathrm{n}}\right)}{\left(1+\frac{\mathrm{m} \phi \mathrm{b}}{2}\right) \tan \left(\beta_{\mathrm{n}}\right)+\frac{1}{2} \beta_{\mathrm{n}}} e^{-\frac{\beta_{\mathrm{n}^{2}}}{\mathrm{~b}} \tau} \\
& b=1-\varepsilon+q \varepsilon \\
& \tau=\frac{\mathrm{D}_{\mathrm{ex}}}{\mathrm{R}^{2}} \mathrm{t} \\
& \phi=\frac{3 \phi^{\prime}}{1-\phi^{\prime}} \\
& \mathrm{m}=\frac{\mathrm{K}_{\mathrm{eq}} \cdot \mathrm{C}_{(\mathrm{RH})_{2} \mathrm{O}^{2}}}{\mathrm{C}_{\mathrm{HO}}^{2}} \\
& \mathrm{q}=\frac{\mathrm{C}_{\mathrm{HOi}}{ }^{2}}{\mathrm{~K}_{\text {eq }} \cdot \mathrm{C}_{(\mathrm{RH})_{2} \mathrm{O}}{ }^{2}} \\
& \beta_{\mathrm{n}} \text { are eigen value of } \tan (\beta)=\frac{\mathrm{m} \phi \mathrm{b} \beta}{\mathrm{m} \phi \mathrm{b}+\beta^{2}}
\end{aligned}
$$

Only the Membrane External Film Mass Transfer Resistance is Considered In this case, the membrane external interphase mass transfer resistance is considered while the membrane internal interphase mass transfer resistance is neglected. The governing equations for the uranium extraction process are: Membrane phase:

$$
(1-\varepsilon) \frac{\partial \mathrm{C}_{\mathrm{x}}}{\partial \mathrm{t}}=\mathrm{D}_{\mathrm{ex}}\left(\frac{1}{\mathrm{r}^{2}} \frac{\partial}{\partial \mathrm{r}}\left(\mathrm{r}^{2} \frac{\partial \mathrm{C}_{\mathrm{x}}}{\partial \mathrm{r}}\right)\right)-\mathrm{R}_{\mathrm{x}}
$$

$$
\begin{aligned}
& \mathrm{t}=0 \mathrm{C}_{\mathrm{X}}=0 \text { for all } \mathrm{r} \\
& \mathrm{r}=0 \mathrm{C}_{\mathrm{X}}=\mathrm{finite}_{\mathrm{N}^{*}} \\
& \mathrm{r}=\mathrm{R} \mathrm{C}_{\mathrm{X}}=\mathrm{C}_{\mathrm{X}}
\end{aligned}
$$


External source phase:

$$
\begin{aligned}
& \quad-\mathrm{V}_{\mathrm{t}}\left(1-\phi^{\prime}\right) \frac{\mathrm{dCu}}{\mathrm{dt}}=\mathrm{N}\left(4 \pi \mathrm{R}^{2}\right) \mathrm{k}_{\mathrm{o}}\left(\mathrm{Cu}-\mathrm{Cu}^{*}\right) \\
& \mathrm{t}=0 \quad \mathrm{Cu}=\mathrm{Cuo}
\end{aligned}
$$

After solving the above equations; considering the boundary conditions, the following mathematical models were obtained:

$$
\begin{aligned}
& \frac{\mathrm{Cu}}{\mathrm{Cuo}}=\frac{3}{3+\mathrm{m} \phi \mathrm{b}}+\sum_{\mathrm{n}=1}^{\infty} \frac{\left(1-\mathrm{mK}_{\mathrm{o}}\right) \tan \left(\beta_{\mathrm{n}}\right)+\mathrm{mK}_{\mathrm{o}} \beta_{\mathrm{n}}}{\left(1-\mathrm{mK}_{\mathrm{o}}-\frac{1}{2} \mathrm{mK}_{\mathrm{o}} \beta_{\mathrm{n}}^{2}+\frac{1}{2} \mathrm{~m} \phi \mathrm{b}\right) \tan \left(\beta_{\mathrm{n}}\right)+\left(\frac{1}{2}+\mathrm{mK}_{\mathrm{o}}\right) \beta_{\mathrm{n}}} \mathrm{e}^{-\frac{\beta_{\mathrm{n}}^{2}}{\mathrm{~b}} \tau} \\
& \mathrm{b}=1-\varepsilon+\mathrm{q} \varepsilon \\
& \tau=\frac{\mathrm{D}_{\mathrm{ex}}}{\mathrm{R}^{2}} \mathrm{t} \\
& \phi=\frac{3 \phi^{\prime}}{1-\phi^{\prime}} \\
& \mathrm{m}=\frac{\mathrm{K}_{\mathrm{eq}} \cdot \mathrm{C}_{(\mathrm{RH})_{2} \mathrm{O}^{2}}}{\mathrm{C}_{\mathrm{HO}}{ }^{2}} \\
& \mathrm{q}=\frac{\mathrm{C}_{\mathrm{HOi}}^{2}}{\mathrm{~K}_{\mathrm{eq}} \cdot \mathrm{C}_{(\mathrm{RH})_{2} \mathrm{O}}^{2}} \\
& \mathrm{~K}_{\mathrm{o}}=\frac{\mathrm{D}_{\mathrm{ex}}}{\mathrm{Rk}_{\mathrm{o}}} \\
& \beta_{\mathrm{n}} \text { are eigen values of } \tan (\beta)=\frac{\left(\mathrm{m} \phi \mathrm{b}-\mathrm{mK}_{\mathrm{o}} \beta^{2}\right) \beta}{\left(1-\mathrm{mK}_{\mathrm{o}}\right) \beta^{2}+\mathrm{m} \phi \mathrm{b}}
\end{aligned}
$$




\section{Both the Membrane External and Internal Film Mass Transfer Resistance are Considered}

In this case both the membrane external interphase mass transfer resistance and the membrane internal interphase mass transfer resistance are considered. The governing eqns. for the uranium extraction process are:

Membrane phase:

$$
\begin{aligned}
& (1-\varepsilon) \frac{\partial \mathrm{C}_{\mathrm{x}}}{\partial \mathrm{t}}=\mathrm{D}_{\mathrm{ex}}\left(\frac{1}{\mathrm{r}^{2}} \frac{\partial}{\partial \mathrm{r}}\left(\mathrm{r}^{2} \frac{\partial \mathrm{C}_{\mathrm{x}}}{\partial \mathrm{r}}\right)\right)-\mathrm{k}_{\mathrm{i}} \mathrm{a}\left(\mathrm{C}_{\mathrm{x}}-\mathrm{C}_{\mathrm{xi}}^{*}\right) \\
\mathrm{t}= & 0 \mathrm{C}_{\mathrm{X}}=0 \text { for all } \mathrm{r} \\
\mathrm{r}= & 0 \mathrm{C}_{\mathrm{X}}=\text { finite } \\
\mathrm{r}= & \mathrm{R} \mathrm{C}_{\mathrm{X}}=\mathrm{C}
\end{aligned}
$$

ExternalC source phase:

$$
\begin{aligned}
& -\mathrm{V}_{\mathrm{t}}\left(1-\phi^{\prime}\right) \frac{\mathrm{dCu}}{\mathrm{dt}}=\mathrm{N}\left(4 \pi \mathrm{R}^{2}\right) \mathrm{k}_{\mathrm{o}}\left(\mathrm{Cu}-\mathrm{Cu}^{*}\right) \\
& \mathrm{t}=0 \quad \mathrm{Cu}=\mathrm{Cuo} \\
& \mathrm{N}\left(4 \pi \mathrm{R}^{2}\right) \mathrm{k}_{\mathrm{o}}\left(\mathrm{Cu}-\mathrm{Cu}{ }^{*}\right)=N\left(4 \pi \mathrm{R}^{2}\right) \mathrm{D}_{\text {ex }}\left(\frac{\partial \mathrm{C}_{\mathrm{x}}}{\partial \mathrm{r}}\right) \mathrm{r}=\mathrm{R}
\end{aligned}
$$

After solving the above equations; considering the boundary conditions, the following mathematical models were obtained:

$$
\begin{aligned}
& \frac{\mathrm{Cu}}{\mathrm{CuO}}=\frac{3}{3+\mathrm{m} \phi \mathrm{b}}+\sum_{\mathrm{n}=1}^{\infty} \frac{\left[\left(1-\mathrm{mK}_{\mathrm{o}}\right) \tan \left(\mathrm{h}_{\mathrm{n}}\right)+\mathrm{mK}_{\mathrm{o}} \mathrm{h}_{\mathrm{n}}\right] \mathrm{e}^{-\beta_{\mathrm{n}} \tau}}{\left[1-\mathrm{mK}_{\mathrm{o}}+\frac{1}{2}\left(\mathrm{~m} \phi-\beta_{\mathrm{n}} \mathrm{mK}_{\mathrm{o}}\right)\left(\frac{\mathrm{h}_{\mathrm{n}}^{2}}{\beta_{\mathrm{n}}}-\mathrm{Q}\left(-\beta_{\mathrm{n}}\right)\right)\right] \tan \left(\mathrm{h}_{\mathrm{n}}\right)+\left(\frac{1}{2}-\frac{\beta_{\mathrm{n}}}{2 \mathrm{~h}_{\mathrm{n}}^{2}} \mathrm{Q}\left(-\beta_{\mathrm{n}}\right)+\mathrm{mK}_{\mathrm{o}}\right) \mathrm{h}_{\mathrm{n}}} \\
& +\sum_{\mathrm{j}=1\left[1\left(1-\mathrm{mK}_{\mathrm{o}}\right) \tan \left(\mathrm{h}_{\mathrm{j}}\right)+\mathrm{mK}_{0} \mathrm{~h}_{\mathrm{j}}\right] \mathrm{e}^{-\gamma_{\mathrm{n}} \tau}} \frac{[28)}{\left[1-\mathrm{mK}_{\mathrm{o}}+\frac{1}{2}\left(\mathrm{~m} \phi-\gamma_{\mathrm{j}} \mathrm{mK}_{\mathrm{o}}\right)\left(\frac{\mathrm{h}_{\mathrm{j}}^{2}}{\gamma_{\mathrm{j}}}-\mathrm{Q}\left(-\gamma_{\mathrm{j}}\right)\right] \tan \left(\mathrm{h}_{\mathrm{j}}\right)+\left(\frac{1}{2}-\frac{\gamma_{\mathrm{j}}}{2 \mathrm{~h} 2} \mathrm{Q}\left(-\gamma_{\mathrm{j}}\right)+\mathrm{mK}_{\mathrm{o}}\right) \mathrm{h}_{\mathrm{j}}\right.}
\end{aligned}
$$


$\mathrm{b}=1-\varepsilon+\mathrm{q} \varepsilon$

$\tau=\frac{\mathrm{D}_{\mathrm{ex}}}{\mathrm{R}^{2}} \mathrm{t}$

$y=\frac{r}{R}$

$\phi=\frac{3 \phi^{\prime}}{1-\phi^{\prime}}$

$\mathrm{K}_{\mathrm{o}}=\frac{\mathrm{D}_{\mathrm{ex}}}{\mathrm{R} \mathrm{K}_{\mathrm{o}}}$

$\mathrm{K}_{\mathrm{i}}=\frac{\mathrm{D}_{\mathrm{ex}}}{\mathrm{R}^{2} \mathrm{k}_{\mathrm{i}} \mathrm{a}}$

$\mathrm{a}=\frac{3 \varepsilon}{\mathrm{d}_{\mathrm{r}}}$

$\mathrm{m}=\frac{\mathrm{K}_{\mathrm{eq}} \cdot \mathrm{C}_{(\mathrm{RH})_{2} \mathrm{O}^{2}}}{\mathrm{C}_{\mathrm{HO}^{2}}}$

$\mathrm{q}=\frac{\mathrm{C}_{\mathrm{HOi}}{ }^{2}}{\mathrm{~K}_{\text {eq }} \cdot \mathrm{C}_{(\mathrm{RH})_{2} \mathrm{O}}{ }^{2}}$

$q(s)=\frac{q^{2} \varepsilon^{2} K_{i} s}{\left(q \varepsilon q_{i} s+1\right)^{2}}$

$\tan (\mathrm{h}) \frac{\left(-\beta m \mathrm{~K}_{\mathrm{o}}+\mathrm{m} \phi\right) \mathrm{h}}{\mathrm{m} \phi+\left(1-\mathrm{mK}_{\mathrm{o}}\right) \beta}$

$\tan (\mathrm{h})=\frac{\left(-\gamma \mathrm{m} \mathrm{K}_{\mathrm{o}}+\mathrm{m} \phi\right) \mathrm{h}}{\mathrm{m} \phi+\left(1-\mathrm{mK}_{\mathrm{o}}\right) \gamma}$ 


$$
\begin{aligned}
& \mathrm{s}^{+}=-\beta=\frac{-\left(\mathrm{b}+\mathrm{q} \varepsilon \mathrm{K}_{\mathrm{i}} \mathrm{h}^{2}\right)+\sqrt{\left(\mathrm{b}+\mathrm{q} \varepsilon \mathrm{K}_{\mathrm{i}} \mathrm{h}^{2}\right)^{2}-4(1-\varepsilon) \mathrm{q} \varepsilon \mathrm{K}_{\mathrm{i}} \mathrm{h}^{2}}}{2(1-\varepsilon) \mathrm{q} \varepsilon \mathrm{K}_{\mathrm{i}}} \\
& \mathrm{s}^{-}=-\gamma=\frac{-\left(\mathrm{b}+\mathrm{q} \varepsilon \mathrm{K}_{\mathrm{i}} \mathrm{h}^{2}\right)+\sqrt{\left(\mathrm{b}+\mathrm{q} \varepsilon \mathrm{K}_{\mathrm{i}} \mathrm{h}^{2}\right)^{2}-4(1-\varepsilon) \mathrm{q} \varepsilon \mathrm{K}_{\mathrm{i}} \mathrm{h}^{2}}}{2(1-\varepsilon) \mathrm{q} \varepsilon \mathrm{K}_{\mathrm{i}}}
\end{aligned}
$$

Solvent Extraction by Liquid Membrane Systems, Uranium and Molybdenum

In this process, liquid membrane is usually in $\mathrm{O} / \mathrm{W} / \mathrm{O}$ form and it can be used for solvent extraction of both uranium and molybdenum. Several assumptions were made to model the complicated batch process of solvent extraction by emulsion liquid membranes.

The governing equations for elements A (uranium) and B (molybdenum), and extract solvent $\mathrm{C}$ in each phase are as follows:

Membrane phase:

$$
\begin{aligned}
& (1-\varepsilon) \frac{\partial \mathrm{C}_{\mathrm{A}}}{\partial \mathrm{t}}=\mathrm{D}_{\mathrm{eA}}\left(\frac{1}{\mathrm{r}^{2}} \frac{\partial}{\partial \mathrm{r}}\left(\mathrm{r}^{2} \frac{\partial \mathrm{C}_{\mathrm{A}}}{\partial \mathrm{r}}\right)\right)+\mathrm{R}_{\mathrm{A}} \\
& \mathrm{t}=\mathrm{t}_{\mathrm{O}} \quad \mathrm{C}_{\mathrm{A}}=\mathrm{C}_{\mathrm{AO}} \\
& \mathrm{r}=0 \quad \mathrm{C}_{\mathrm{A}}=\text { finite } \\
& \mathrm{r}=\mathrm{R} \quad \mathrm{C}_{\mathrm{A}}=\mathrm{C}^{\mathrm{A}} \\
& (1-\varepsilon) \frac{\partial \mathrm{C}_{\mathrm{B}}}{\partial \mathrm{t}}=\mathrm{D}_{\mathrm{eB}}\left(\frac{1}{\mathrm{r}^{2}} \frac{\partial}{\partial \mathrm{r}}\left(\mathrm{r}^{2} \frac{\partial \mathrm{C}_{\mathrm{B}}}{\partial \mathrm{r}}\right)\right)+\mathrm{R}_{\mathrm{B}} \\
& \mathrm{t}=\mathrm{t}_{\mathrm{O}} \quad \mathrm{C}_{\mathrm{B}}=\mathrm{C}_{\mathrm{BO}} \\
& \mathrm{r}=0 \quad \mathrm{C}_{\mathrm{B}}=\text { finite } \\
& \mathrm{r}=1 \quad \mathrm{C}_{\mathrm{B}}=\mathrm{C}_{\mathrm{B}}^{*} \\
& (1-\varepsilon) \frac{\partial \mathrm{C}_{\mathrm{C}}}{\partial \mathrm{t}}=\mathrm{D}_{\mathrm{eC}}\left(\frac{1}{\mathrm{r}^{2}} \frac{\partial}{\partial \mathrm{r}}\left(\mathrm{r}^{2} \frac{\partial \mathrm{C}_{\mathrm{C}}}{\partial \mathrm{r}}\right)\right)+\mathrm{R}_{\mathrm{C}} \\
& \mathrm{t}=\mathrm{t}_{\mathrm{O}} \quad \mathrm{C}_{\mathrm{C}}=\mathrm{C}_{\mathrm{CO}} \\
& \mathrm{r}=0 \quad \mathrm{C}_{\mathrm{C}}=\text { finite } \\
& \mathrm{r}=\mathrm{R} \quad \mathrm{C}_{\mathrm{C}}=\mathrm{C}_{\mathrm{C}}^{*}
\end{aligned}
$$

External extract phase:

$$
\begin{aligned}
& \frac{d A}{d t}=-N\left(4 \pi R^{2}\right) D_{e A}\left(\frac{\partial C_{A}}{\partial r}\right)_{r=R} \\
t= & t_{O} \quad A=A_{O}
\end{aligned}
$$




$$
\begin{aligned}
& \frac{\mathrm{dB}}{\mathrm{dt}}=-\mathrm{N}\left(4 \pi \mathrm{R}^{2}\right) \mathrm{D}_{\mathrm{eB}}\left(\frac{\partial \mathrm{C}_{\mathrm{A}}}{\partial \mathrm{r}}\right)_{\mathrm{r}=\mathrm{R}} \\
\mathrm{t}= & \mathrm{t}_{\mathrm{O}} \quad \mathrm{B}=\mathrm{B}_{\mathrm{O}} \\
& \frac{\mathrm{dC}}{\mathrm{dt}}=-\mathrm{N}\left(4 \pi \mathrm{R}^{2}\right) \mathrm{D}_{\mathrm{eC}}\left(\frac{\partial \mathrm{C}_{\mathrm{C}}}{\partial \mathrm{r}}\right)_{\mathrm{r}=\mathrm{R}} \\
\mathrm{t}= & \mathrm{t}_{\mathrm{O}} \quad \mathrm{C}=\mathrm{C}_{\mathrm{O}} \\
\mathrm{N}= & \frac{\mathrm{V}_{\mathrm{EO}}}{(4 / 3) \pi \mathrm{R}_{\mathrm{O}}^{3}}
\end{aligned}
$$

Internal raffinate phase:

$$
\begin{aligned}
& \varepsilon \mathrm{M}_{\mathrm{R}} \frac{\mathrm{X}_{\mathrm{A}}}{\mathrm{t}}=-\mathrm{R}_{\mathrm{A}} \\
& \mathrm{t}=\mathrm{t}_{\mathrm{O}} \quad \mathrm{X}_{\mathrm{A}}=\mathrm{X}_{\mathrm{AO}} \\
& \varepsilon \mathrm{M}_{\mathrm{R}} \frac{\mathrm{X}_{\mathrm{B}}}{\mathrm{t}}=-\mathrm{R}_{\mathrm{B}} \\
& \mathrm{t}=\mathrm{t}_{\mathrm{O}} \quad \mathrm{X}_{\mathrm{B}}=\mathrm{X}_{\mathrm{BO}} \\
& \quad \varepsilon \mathrm{M}_{\mathrm{R}} \frac{\mathrm{X}_{\mathrm{C}}}{\mathrm{t}}=-\mathrm{R}_{\mathrm{C}} \\
& \mathrm{t}=\mathrm{t}_{\mathrm{O}} \quad \mathrm{X}_{\mathrm{C}}=\mathrm{X}_{\mathrm{CO}}
\end{aligned}
$$

Having the above parameter values, we can calculate $\mathrm{A}, \mathrm{B}$ and $\mathrm{C}$ at next short time $t=t_{1}$. When we have the moles of $A, B$ and $C$ at time $t_{1}$, we can adjust the parameter values as follows:

$$
\begin{aligned}
& C_{A O}=m_{A} M_{m} X_{A}=m_{A} M_{m} \frac{A_{O i}-A}{A_{O i}+B_{O i}-A-B+C} \\
& C_{B O}=m_{B} M_{m} X_{B}=m_{B} M_{m} \frac{B_{O i}-B}{A_{O i}+B_{O i}-A-B+C} \\
& C_{C O}=m_{C} M_{m} X_{C}=m_{C} M_{m} \frac{C}{A_{O i}+B_{O i}-A-B+C}
\end{aligned}
$$




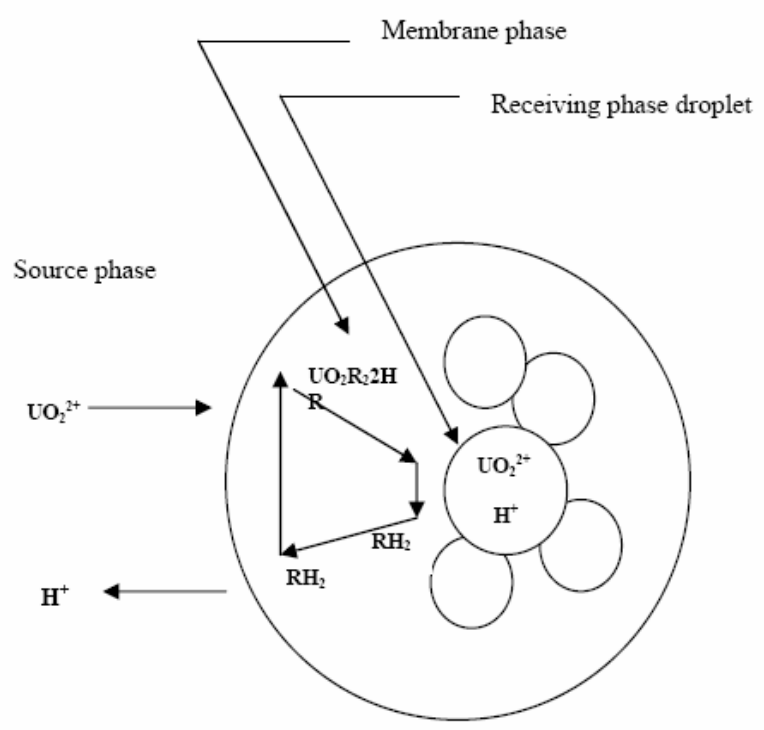

A globule in a batch

Fig. 2. Mechanism of Carrier Mediated Uranium Extraction through Emulsion Liquid Membranes

\section{RESULTS AND DISCUSSION}

For both uranium and molybdenum extraction modeling process, several mathematical models have been developed in this research. For different cases of modeling, such as (1) when the internal film resistance is negligible:

$$
\begin{aligned}
& \frac{\mathrm{Cu}}{\mathrm{Cuo}}=\lim _{\mathrm{K}_{\mathrm{i}} \rightarrow 0}\left[\frac{3}{3+m \phi b}\right]+\left[\sum_{\mathrm{n}=1}^{\infty} \frac{\left[\left(\mathrm{i}-\mathrm{mK}_{\mathrm{o}}\right) \tan \left(\mathrm{h}_{\mathrm{n}}\right)+\mathrm{mK}_{\mathrm{o}} \mathrm{h}_{\mathrm{n}}\right] \mathrm{e}^{-\beta_{\mathrm{n}} \tau}}{\left.\mathrm{m} \mathrm{K}_{\mathrm{o}}+\frac{1}{2}\left(\mathrm{~m} \varphi-\beta_{\mathrm{n}} \mathrm{mK} \mathrm{K}_{\mathrm{o}}\right)\left(\frac{\mathrm{h}_{\mathrm{n}}}{\beta_{\mathrm{n}}}-\mathrm{Q}\left(-\beta_{\mathrm{n}}\right)\right)\right] \tan \left(\mathrm{h}_{\mathrm{h}}\right)+\left(\frac{1}{2}-\frac{\mathrm{n}}{2 \mathrm{~h}_{\mathrm{n}}^{2}} \mathrm{Q}\left(-\beta_{\mathrm{n}}\right)+\mathrm{mK}_{\mathrm{o}}\right) \mathrm{h}_{\mathrm{n}}}\right] \\
& +\left[\sum_{\mathrm{n}=1}^{\infty} \frac{\left(1-\mathrm{mK}_{\mathrm{o}}\right) \tan \left(\mathrm{h}_{\mathrm{j}}\right)+\mathrm{mK}_{\mathrm{o}} \mathrm{h}_{\mathrm{j}} \mathrm{e}^{-\gamma_{\mathrm{j}}}}{1-\mathrm{mK}_{\mathrm{o}}+\frac{1}{2}\left(\mathrm{~m} \phi-\gamma_{\mathrm{j}} \mathrm{mK}_{\mathrm{o}}\right)\left(\frac{\mathrm{h}_{\mathrm{j}}}{\gamma_{\mathrm{j}}}-\mathrm{Q}\left(-\gamma_{\mathrm{j}}\right)\right) \tan \left(\mathrm{h}_{\mathrm{j}}\right)+\left(\frac{1}{2}-\frac{\gamma_{\mathrm{j}}}{2 \mathrm{~h}_{\mathrm{j}}^{2}} \mathrm{Q}\left(-\gamma_{\mathrm{j}}\right)+\mathrm{mK}_{\mathrm{o}} \mathrm{h}_{\mathrm{j}}\right.}\right] \frac{3}{3+\mathrm{m} \phi \mathrm{b}} \\
& +\sum_{\mathrm{n}=1\left(1-\mathrm{mK}_{\mathrm{o}}-\frac{1}{2} \mathrm{mK}_{\mathrm{o}} \mathrm{h}_{\mathrm{n}}^{2}+\frac{1}{2} \mathrm{mb}\right) \tan \left(\mathrm{h}_{\mathrm{n}}\right)+\left(\frac{1}{2}+\mathrm{mK}_{\mathrm{o}}\right) \mathrm{h}_{\mathrm{n}}} \mathrm{e}^{-\frac{\mathrm{h}_{\mathrm{n}}^{2}}{\mathrm{~b}}}
\end{aligned}
$$

(2) when the external film resistance is negligible: 


$$
\begin{aligned}
& \frac{\mathrm{Cu}}{\mathrm{Cuo}}=\lim _{\mathrm{K}_{\mathrm{o}} \rightarrow 0}\left[\frac{3}{3+\mathrm{m} \phi \mathrm{b}}+\sum_{\mathrm{n}=1}^{\infty} \frac{\left(1-\mathrm{mK}_{\mathrm{o}}\right) \tan \left(\beta_{\mathrm{n}}\right)+\mathrm{mK}_{\mathrm{o}} \beta_{\mathrm{n}}}{\left.\frac{\mathrm{m}}{2} \mathrm{KK}_{\mathrm{o}} \beta_{\mathrm{n}}^{2}+\frac{\mathrm{m} \phi \mathrm{b}}{2}\right) \tan \left(\beta_{\mathrm{n}}\right)+\left(\frac{1}{2}+\mathrm{mK}_{\mathrm{o}}\right) \beta_{\mathrm{n}}} \mathrm{e}^{-\frac{\beta_{\mathrm{n}}^{2}}{\mathrm{~b}}}\right] \frac{3}{3+\mathrm{m} \phi \mathrm{b}} \\
& +\sum_{\mathrm{n}=1}^{\infty} \frac{\tan \left(\beta_{\mathrm{n}}\right)}{\left(1+\frac{1}{2} \mathrm{~m} \phi \mathrm{b}\right) \tan \left(\beta_{\mathrm{n}}\right)+\frac{1}{2} \beta_{\mathrm{n}}} \mathrm{e}^{-\frac{\beta_{\mathrm{n}}^{2}}{\mathrm{~b}}}
\end{aligned}
$$

(3) solvent extraction:

$$
\begin{aligned}
& C_{A O}=m_{A} M_{m} X_{A}=m_{A} M_{m} \frac{A_{O i}-A}{A_{O i}+B_{O i}-A-B+C} \\
& C_{B O}=m_{B} M_{m} X_{B}=m_{B} M_{m} \frac{B_{O i}-B}{A_{O i}+B_{O i}-A-B+C} \\
& C_{C O}=m_{C} M_{m} X_{C}=m_{C} M_{m} \frac{C}{A_{O i}+B_{O i}-A-B+C}
\end{aligned}
$$

The mean diameter of globules and equilibrium constant were measured before uranium extraction. Diffusivities and mass transfer coefficients were estimated. The experimental conditions and all parameter values are summarized in Table 1 and Table 2. Having all the parameter values, with the aid of computer software (Mathematica 5.0), the above models will then be used to calculate the external phase uranium concentrations for different cases, and the results are shown in Figures 3 and 4.

Table.1. Experimental Conditions for Uranium Extraction

\begin{tabular}{lcc}
\hline & Run 1 & Run 2 \\
\hline Vol. of membrane phase, $\mathrm{ml}$ & 40 & 40 \\
Vol. of receiving phase, $\mathrm{ml}$ & 40 & 40 \\
Vol. of source phase, $\mathrm{ml}$ & 460 & 460 \\
Con. of $\mathrm{UO}_{2}$ in source phase, ppm & 130 & 174 \\
Con. of $\mathrm{HNO}_{3}$ in Rec. phase, \%w & 1.1 & 1.1 \\
Mixing speed, rpm & 280 & 280 \\
pH value in Rec. phase & 0.77 & 0.77 \\
pH value in source phase & 0.23 & 0.23 \\
\hline
\end{tabular}


Table.2. Summarized Parameters Values for Uranium Extraction

\begin{tabular}{lcc}
\hline & Run 1 & Run 2 \\
\hline$\varepsilon$ & 0.5 & 0.5 \\
$\phi^{\prime}$ & 0.148 & 0.148 \\
$\phi$ & 0.522 & 0.522 \\
$\mathrm{q}$ & 2169 & 2169 \\
$\mathrm{~m}$ & 0.402 & 0.402 \\
$\mathrm{D}_{\mathrm{x}}, \mathrm{cm}^{2} / \mathrm{sec}$ & $7 \times 10^{-7}$ & $7 \times 10^{-7}$ \\
$\mathrm{D}_{\mathrm{UO} 2}$ (in kerosene) & $3.5 \times 10^{-6}$ & $3.5 \times 10^{-6}$ \\
$\mathrm{R}, \mathrm{cm}$ & 0.045 & 0.045 \\
$\mathrm{~d}_{\mathrm{r}}, \mathrm{cm}$ & 0.0004 & 0.0004 \\
$\mathrm{k}_{\mathrm{o}}, \mathrm{cm} / \mathrm{sec}$ & 0.0017 & 0.0017 \\
$\mathrm{k}_{\mathrm{i}}, \mathrm{cm} / \mathrm{sec}$ & 0.0035 & 0.0035 \\
\hline
\end{tabular}

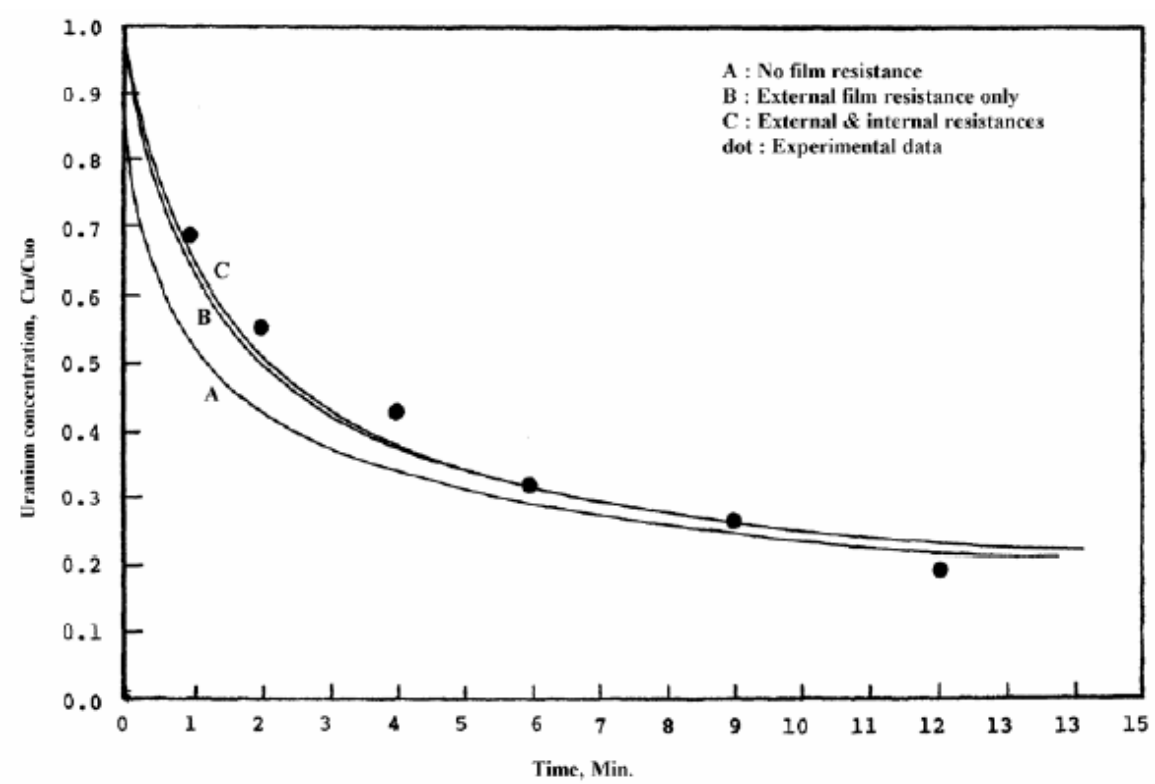

Fig. 3. Uranium Extraction - Run 1 


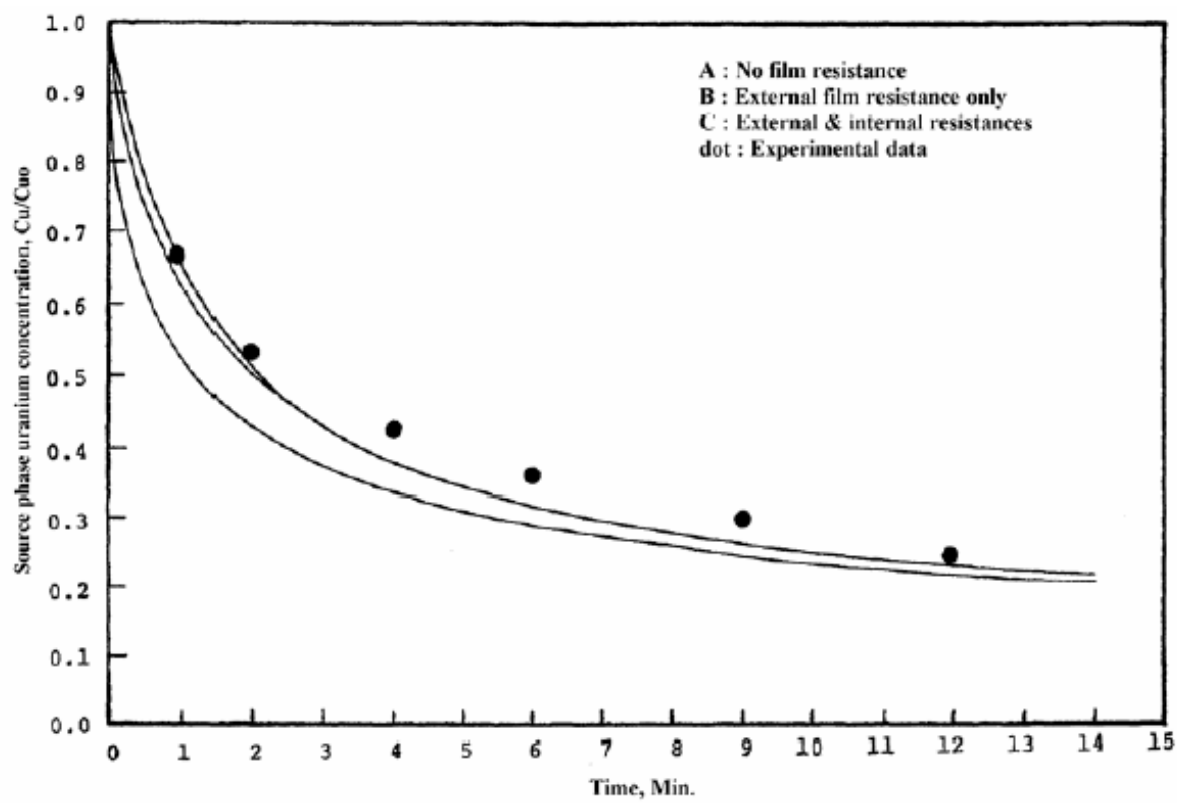

Fig. 4. Uranium Extraction - Run 2

Figure 5 shows the predictions and data for the mass fractions of A (uranium) and B (molybdenum) as a function of time. It can be seen from the Figure, when parameters were adjusted for every 0.125 hour, the predicted mass fraction of uranium in the extract phase is very close to the experimental date while the predicted mass fraction of molybdenum shows some deviation from data. For the curves indicated by $\Delta \mathrm{t}=0.25$ shows some different results.

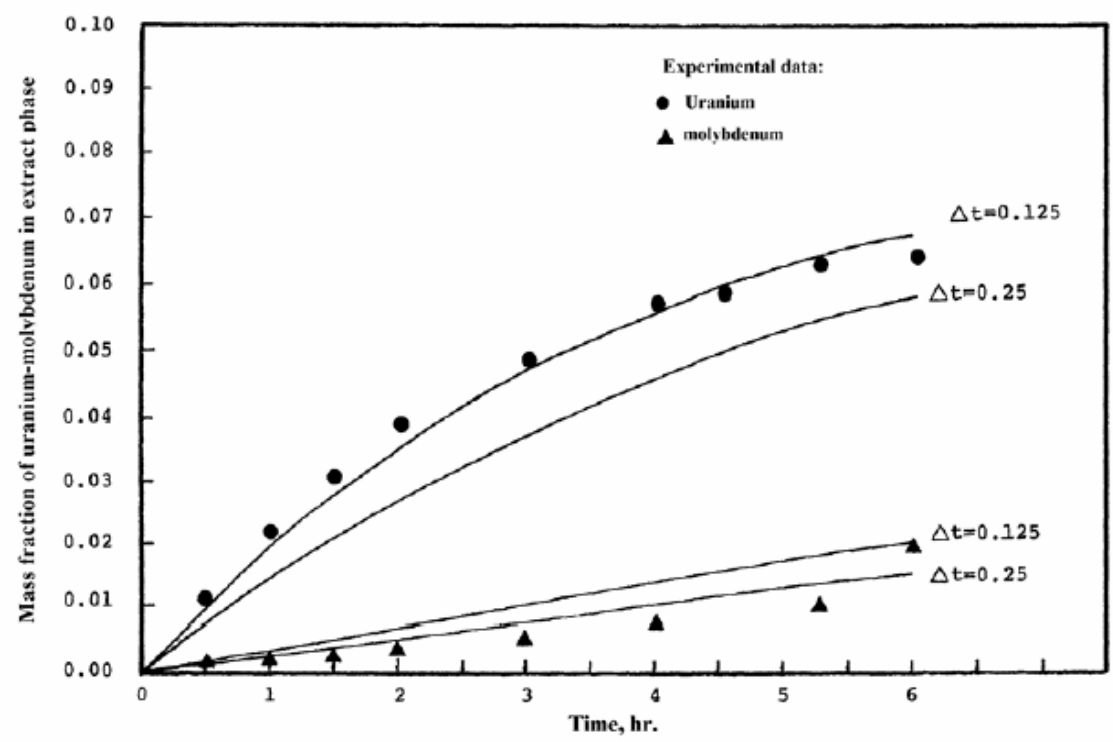

Fig. 5. Solvent Extraction 


\section{Leakage Rate}

From the data, it seems that most of the leakage came at the time when the emulsion phase was breaking into small globules. After that initial period the leakage seems very slow. In practical applications, the $1 \%$ leakage rate is tolerable and is negligible

\section{Effect of Carrier Concentration}

From Figure 6 it can be said that at low carrier concentration, the increase of carrier concentration will increase the extraction rate. After certain optimum concentration, the increase of carrier concentration will have less effect on the uranium extraction rate.

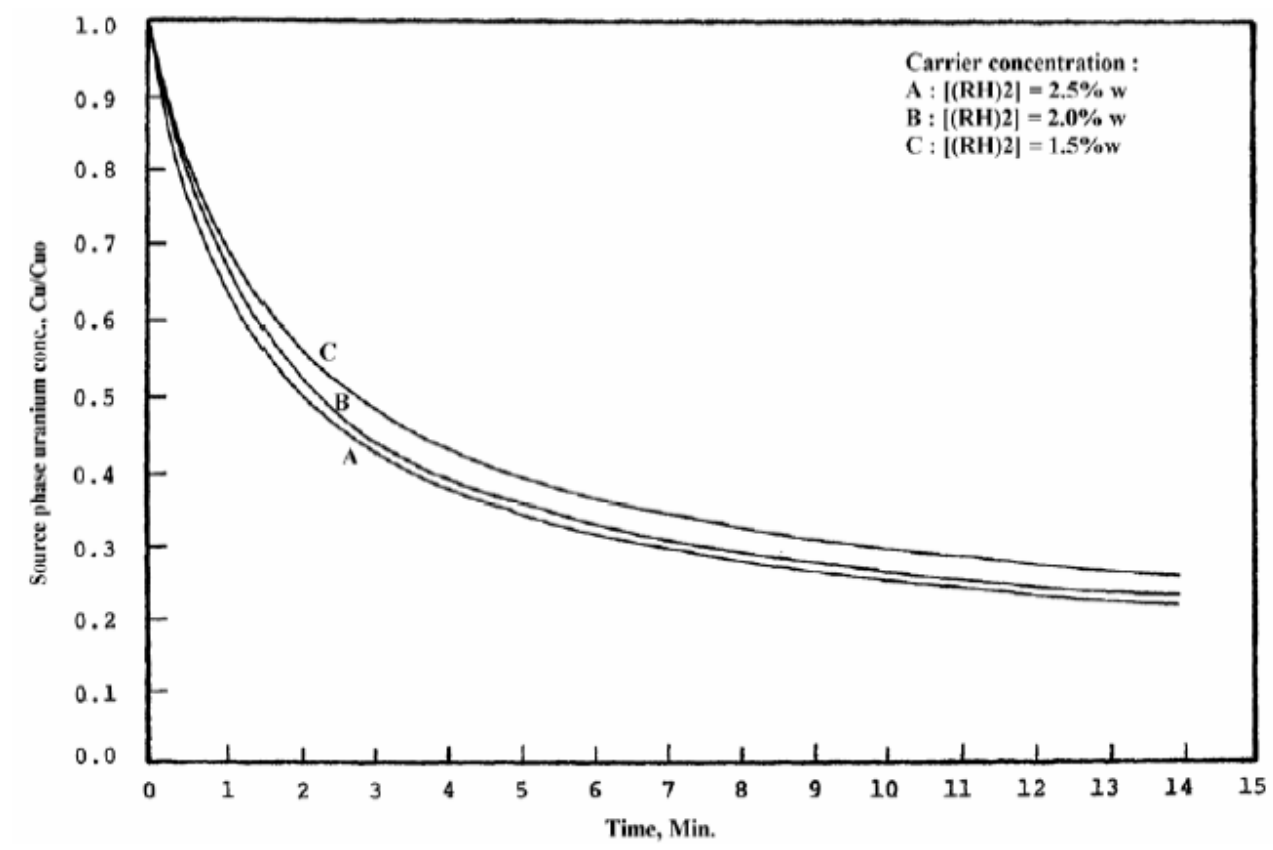

Fig. 6. Effect of Carrier Concentration on Uranium Extraction Rate

\section{Effect of Receiving Phase Nitric Acid Concentration}

Figure 7 shows the effect of receiving phase nitric acid concentration on uranium extraction rate. If the nitric acid concentration is high, then the driving force between the membrane and the receiving phase will also be high, and so the extraction rate will be increased. But since the membrane rupture problem exists, the nitric acid concentration cannot be too high. Too much acid in the external aqueous solution will reduce the effectiveness of the liquid membrane systems. 


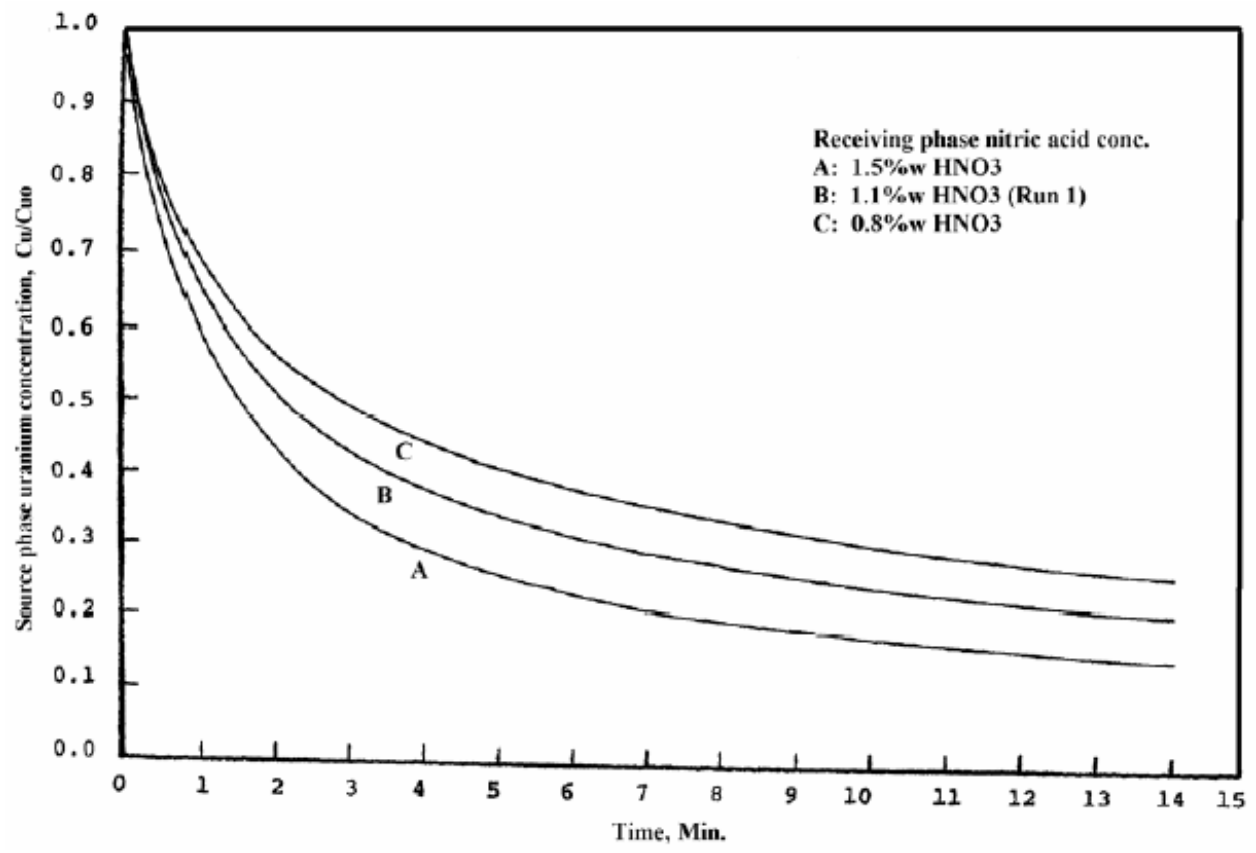

Fig. 7. Effect of Receiving Phase Concentration on Uranium Extraction Rate

\section{Effect of Surfactant Concentration}

Figure 8 shows the effect of Span 80 concentration on the molybdenum extraction rate. As shown in the Figure, higher surfactant concentration results in higher molybdenum extraction rate. But too much surfactant is not desirable, since it will cause some difficulty in breaking emulsion phase in a subsequent process.

\section{Effect of receiving phase $\mathrm{NaOH}$ concentration}

The effect of $\mathrm{NaOH}$ concentration on the molybdenum extraction rate is similar to the nitric acid conc. on the uranium extraction rate. But again, because the leakage problem, the receiving phase $\mathrm{NaOH}$ concentration cannot be too high. Figure 9 shows the effect of $\mathrm{NaOH}$ concentration on the molybdenum extraction rate. 


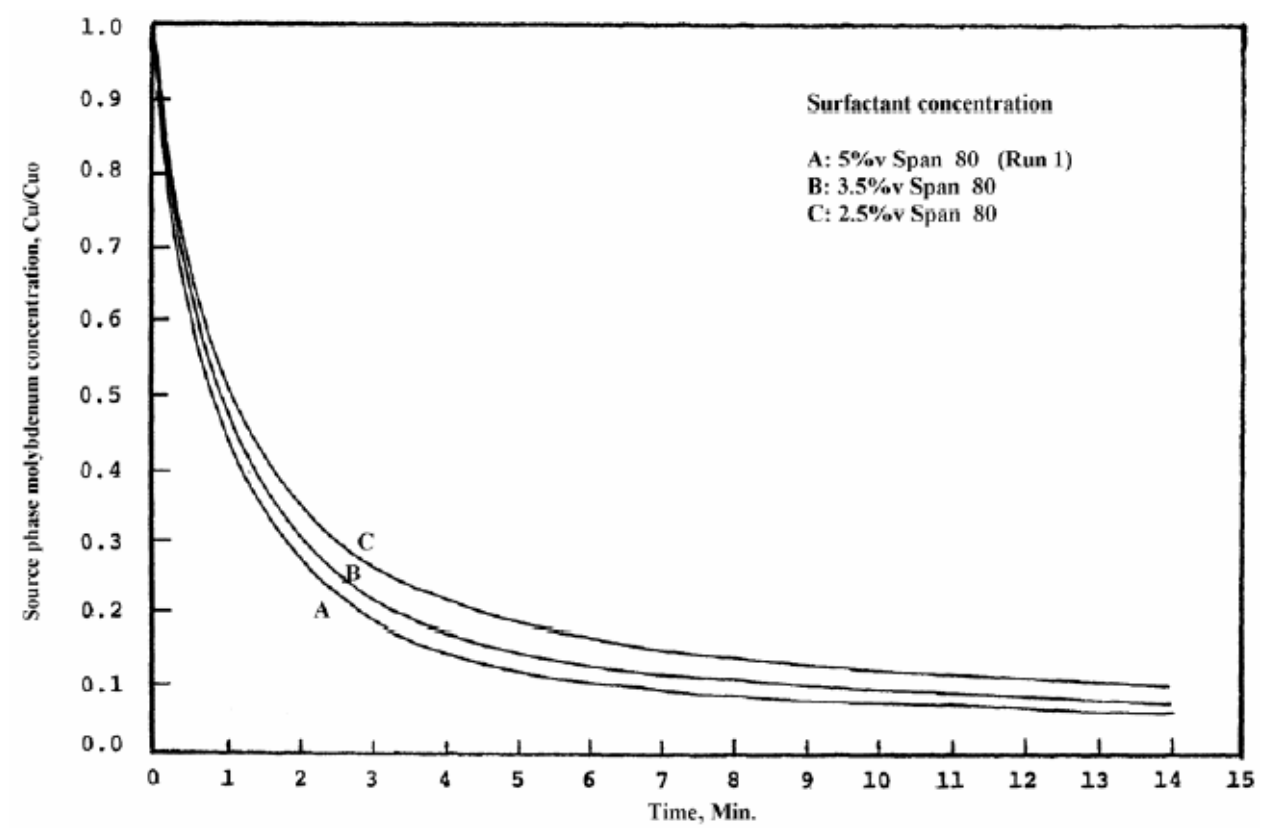

Fig. 8. Effect of Surfactant Concentration on Molybdenum Extraction Rate

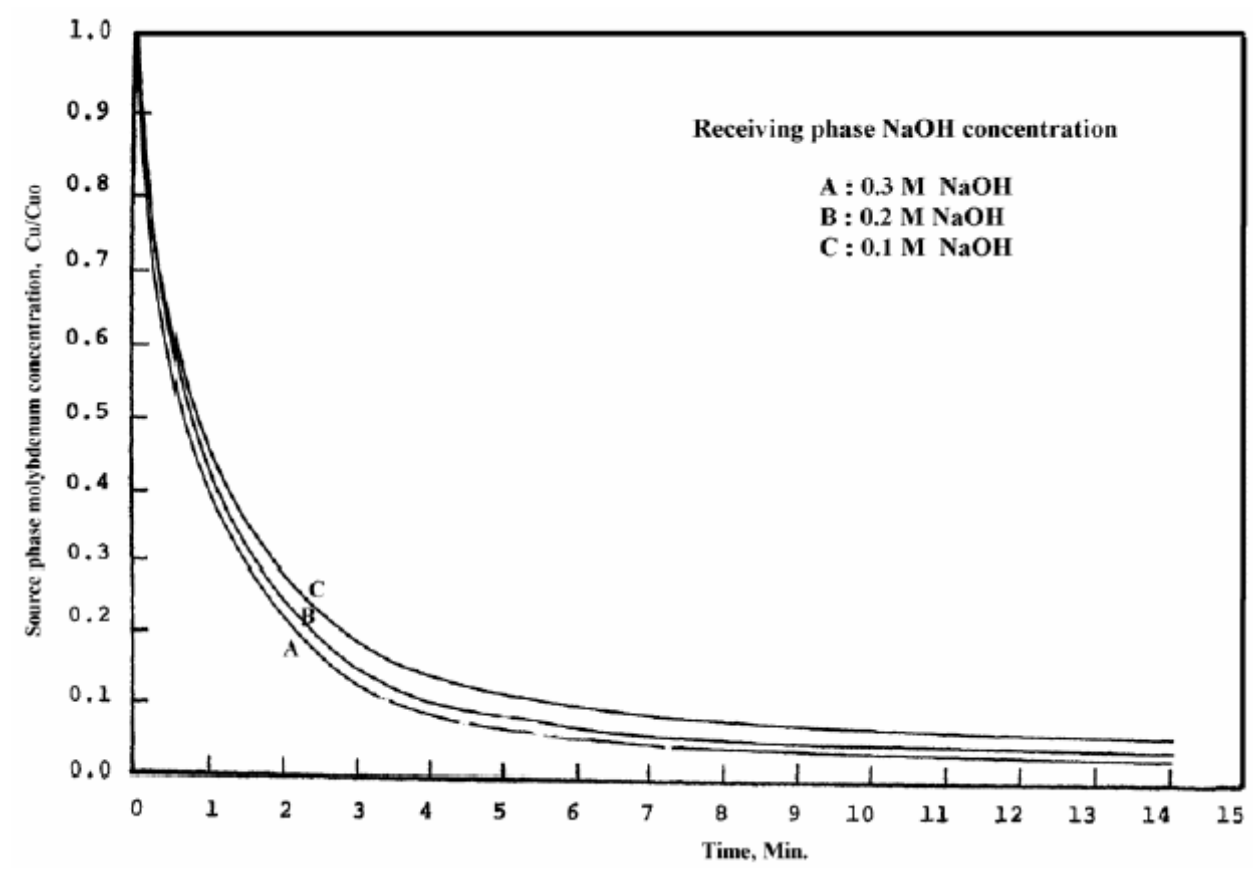

Fig. 9. Effect of Receiving Phase $\mathrm{NaOH}$ Concentration on Molybdenum Extraction Rate 


\section{Cost Comparison of Uranium Recovery by an Emulsion Liquid Membrane and a Traditional Method}

Emulsion liquid membrane systems have the following possible advantages over some traditional methods:

1. Save energy.

2. Reduce cost.

3. Reduce solvent consumption.

4. Produce very high concentrated solution from dilute solution.

This section provides the economic comparison of uranium recovery by an emulsion liquid membrane system and the method current in practice.

In pretreatment, Emulsion Liquid Membrane (ELM) requires no soluble organic removal or cooling, while SX may require some of these steps. In extraction, there is a maximum of three ELM stages versus eight SX stages for extraction and stripping. ELM, however, requires a separate coalescer and emulsifier-offsetting to some extent the capital cost savings associated with the elimination of the stripping stages. The secondary solvent step is identical for both processes.

Table (3) shows the operating costs for the extraction facilities expressed in dollars per pound of uranium recovered. One of the major differences between the ELM and SX costs are associated with organic make-up. The main differences between emulsion liquid membranes and solvent extraction are shown in Table 4. In pretreatment, ELM requires no soluble organic removal or cooling, while SX may require some of these steps. In extraction, there is a maximum of three ELM stages versus eight SX stages for extraction and stripping. ELM, however, requires a separate coalescer and emulsifier-offsetting to some extent the capital cost savings associated with the elimination of the stripping stages. The secondary solvent step is identical for both processes.

Table. 3. Capital Cost Estimates (Basis: 400,000 tons/yr Acid Capacity, 350,000 lbs/yr $\mathrm{U}_{3} \mathrm{O}_{8}$ Recovery, 2nd Qtr 1979)

\begin{tabular}{|c|c|c|c|}
\hline \multicolumn{4}{|c|}{ Dollar per $\mathrm{lb}$ of $\mathrm{U}_{3} \mathrm{O}_{8}$} \\
\hline & \multirow[b]{2}{*}{ ELM } & \multicolumn{2}{|c|}{ SX } \\
\hline & & $\begin{array}{c}\text { Minimum } \\
\text { Pretreatment }\end{array}$ & $\begin{array}{c}\text { Extensive } \\
\text { Pretreatment }\end{array}$ \\
\hline \multicolumn{4}{|l|}{ Organic make-up } \\
\hline Circulation loss & 0.1 & 3.9 & 3.9 \\
\hline Raffinate loss & 0.1 & 0.2 & 0.2 \\
\hline Crud loss and treatment & 1.0 & 2.9 & 0.0 \\
\hline Chemical \& Supplies & 1.4 & 0.9 & 1.6 \\
\hline Utilities & 0.7 & 0.9 & 1.0 \\
\hline Labor, maintenance, tax, $\&$ insurance & 7.1 & 7.1 & 8.6 \\
\hline Depreciation & 4.6 & 4.7 & 5.6 \\
\hline Total operation cost & 15.0 & 20.6 & 20.9 \\
\hline
\end{tabular}


Table. 4. Differences between ELM and Solvent Extraction

\begin{tabular}{lll}
\hline & \multicolumn{1}{c}{ ELM } & \multicolumn{1}{c}{ SX } \\
\hline Feed Pretreatment & $\begin{array}{l}\text { Oxidation } \\
\text { Solid removal }\end{array}$ & $\begin{array}{l}\text { Oxidation } \\
\text { Solid removal } \\
\text { Cooling } \\
\text { Soluble organic removal } \\
\text { Eight stages }\end{array}$ \\
Extraction/stripping & $\begin{array}{l}\text { Max. } 3 \text { stages } \\
\text { Coalescer } \\
\text { Emulsifier }\end{array}$ & \\
& Identical for both & 1 \\
Secondary extraction & $1 / 5$ & 1 \\
$\mathrm{D}_{2}$ EHPA/TOPO conc. & $1 / 4-1 / 5$ & $1: 1$ \\
Crud make & $18: 1$ & \\
Feed/organic ratio & & \\
\hline
\end{tabular}

Block diagram of Uranium Extraction (Both the Membrane External and Internal Film Mass Transfer Resistances are Considered) shown in Fig. 10

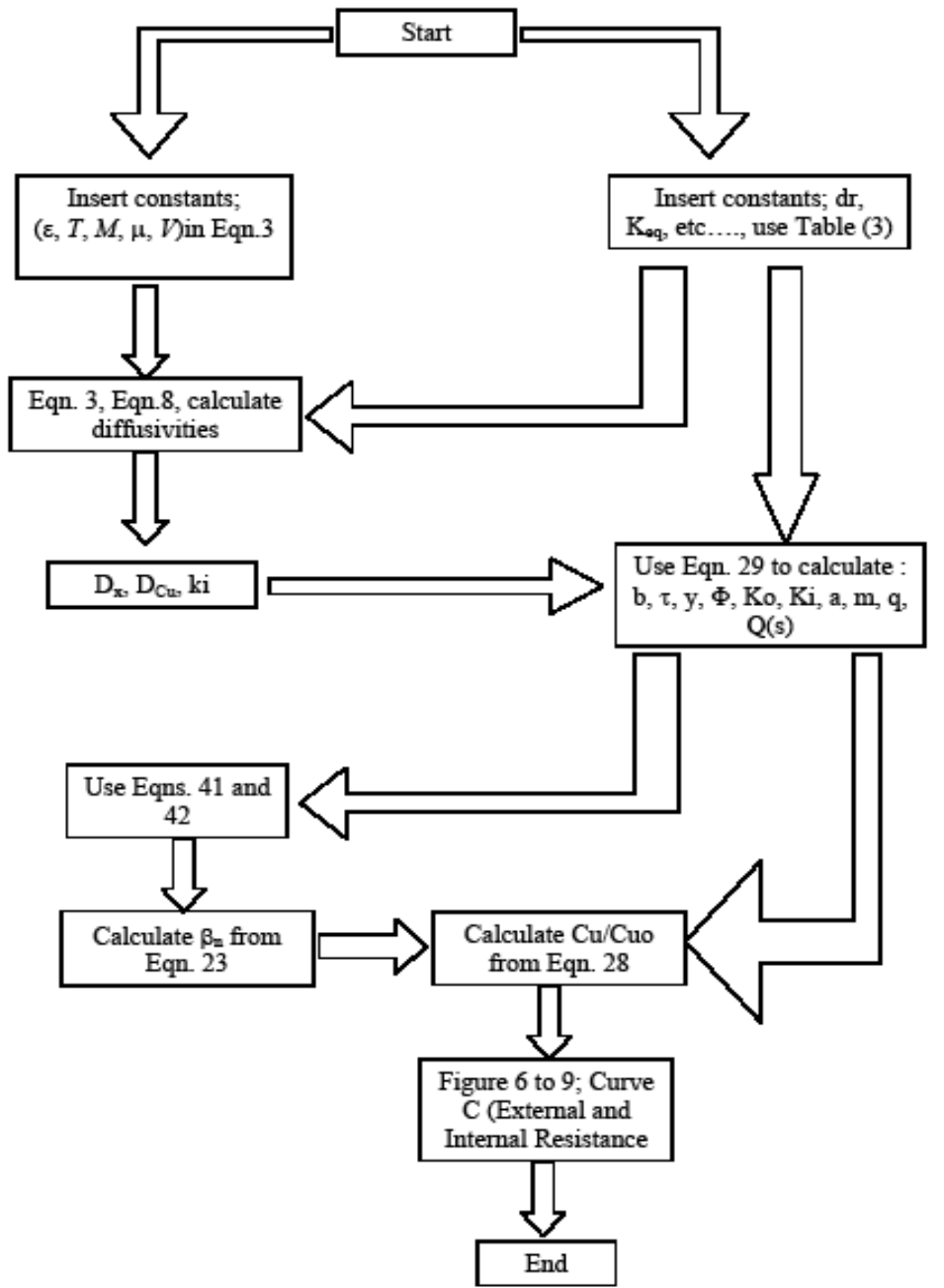

Fig. 10. Block Diagram of Uranium Extraction 


\section{CONCLUSION}

The agreements of the theoretical predictions for the uranium extraction, molybdenum extraction and solvent extraction with experimental data are very good. A very important contribution of this research is that it can predict the extraction rate of an emulsion liquid membrane system without the need of experimental extraction run.

Span 80 is added to membrane phase to stabilize the emulsion and to reduce membrane breakage. From some experiments taken from the literature, at $5 \% \mathrm{v}$ Span 80 , the leakage rate was below $1 \%$. In practical applications, the leakage problem can be neglected. Too high surfactant concentration should be avoided. Since too much surfactant in membrane phase would cause some problems in breaking up the emulsion in a subsequent process.

When chemical reaction is involved in the receiving phase, increase the receiving phase reagent concentration will also increase the extraction rate. Since the membrane rupture problem exists, the receiving phase reagent concentration cannot be too high.

From the comparison of the models with experimental data, it was concluded that for practical purpose, the internal film resistance is negligible and the external film resistance cannot be neglected. The models developed in this research are for batch process, but they can easily be modified for a continuous process. The ELM technology appears to have sufficient return on capital at today's uranium prices to warrant extraction from phosphoric acid plants.

\section{A KNOWLEGMENT}

This research was carried out during $\mathrm{PhD}$ programme of Mr. Haidar and sincerely thanks are extended to Prof. Prayoto and Prof. Harjono the University of Gadjahmada Yogyakarta for usefull discussion.

\section{REFERENCES}

AHN, B.S., LEE, S.C., and Lee, W.K., 1996, "Mathematical Modeling of Silver Extraction by an Emulsion Liquid Membrane Process", J. Membrane Sci., 114, 171-185.

BARTSCH, R.A. and WAY, J.D., 1996, "Chemical Separation with Liquid Membrane", ACS Symposium Series 642, pp. 91-93, 361-372, 181-193, American Chemical Society, California.

BIRD, R.B., STEWART, W.E., LIGHTFOOT, E.N., 1960, Transport Phenomena, pp. 495 515, $594-619$, John Wiley \& Sons, Inc. New York.

BROMBERG, L., LEWIN, I., and WARSHAWSKY, A., 1992, "Membrane Extraction of Silver by Di (2-ethylhexyl) Dithiophosphoric Acid", J. Membrane Sci., 70, 3139. 
CHURCHILL, R. V., 1972, Operational Mathematics, pp. 125 - 164, McGraw-Hill Book Company, New York.

DHADKE, P.M. and SINGH, R.K., 2002, "Extraction and separation of titanium (IV) with D2EHPA and PC-88A from aqueous perchloric acid solutions", J. Serb. Che. Soc., 67, $507-521$.

HAYWORTH, H.C. AND BURNS, W.A., 1983, "Extraction of Uranium from Wet Process Phosphoric Acid by Liquid Membranes", Sep. Sci. Technol., 18, 493521.

HIRAI, T., HASHIMOTO, T., TSUBOI, I, HINO, A., and KOMASAWA, I., 1995, "Extraction and Separation of Molybdenum and Vanadium Using Bis(2Ethylhexyl) Monothiophosphoric Acid and Bis(2-Ethylhexyl) Phosphoric Acid", J. Chem. Eng. Jap., 28, 85 - 90.

HIRAI, T., NISHIHAMA, S., and KOMASAWA, I., 1999, "Mechanism of Photoreductive Stripping of Iron (III) in a Liquid-Liquid Extraction System and Its Application for a Hydrometallurgical Process", Ind. Eng. Chem. Res., $38,4850-4856$.

INCROPERA, F.P. and WITT, P.D., 1990, Fundamentals of Heat and Mass Transfer, 3 ed., pp. A25, John Wiley \& Sons, New York.

JYH-HERNG, C., YING-YUN, K., and CHIA-HUNG, L., 2003, "Selective Separation of Vanadium from Molybdenum Using $\mathrm{D}_{2}$ EHPA-Immobilized Amberlite XAD-4 Resin”, Sep. Sci. Technol., 38, 3827 - 3852.

KHORFAN, S., SHINO, O., WAHOUD, A., and DAHDOUH, A., 2000, "Stripping of Uranium from D2EHPA/TOPO Solvent By Ammonium Carbonate Solutions", Chem. Eng., 44, $123-132$.

KHORFAN, S., SHINO, O., WAHOUD, A., and DAHDOUH, A., 2000, "Stripping of Uranium from D2EHPA/TOPO Solvent By Ammonium Carbonate Solutions", Chem. Eng., $\underline{44}, 123-132$

KRIS TRI BASUKI, 1993, “Kimia Aktinida dalam Teknologi Nuklir”, pp. 3-23, 37-50, Duta wacana University Press., Yogyakarta.

LEE, Y.K., MOK, Y.S., and LEE, W.K., 1997, "Modeling of Liquid Emulsion Membranes Facilitated by Two Carries", Chem. Eng. J., 66, 11-20.

LIU, X. and ZHANG, X., 1997, "Simplified Model for Extraction of Rare-Earth Ions Using Emulsion Liquid Membrane”, J. Membrane Sci., 128, 223-229.

MORRISON, S.J., and SPANGLER, R.R., 1992, "Extraction of Uranium and Molybdenum from Aqueous Solutions: A Survey of Industrial Materials for Use in Chemical Barriers for Uranium Mill Tailings Remediation", Environ. Sci. Technol., 26, $1922-1931$.

MULDER, M., 1996, "Basic Principles of Membrane Science”, 2 ed., pp. 12, 340-342, Kluwer Academic Publishers, Dordrecht.

ÖZIŞIK, M.N., 1985, Heat Transfer: A Basic Approach, pp. 720 - 723, McGraw-Hill, Inc., New York. 
PERRY, R.H. and GREEN, D.W., 1997, Perry's Chemical Engineerings' Handbook, 7 ed., pp. 2-19, 2-26, 2-152, 2-152, 2-330, 2-372, McGraw-Hill, Inc., New York.

REINHOLD, V. N., 1992, Membrane Handbook, pp. 597-610, 611-655, 656-700,701717, McGraw-Hill, Inc., New York.

TING-CHIA, H. and CHING-TSVEN, H., 1988, "Kinetics of the Extraction of Uranium (VI) from Nitric Acid Solution with Bis (2-ethylhexyl) Phosphoric Acid", Ind. Eng. Chem. Res., 27, 1675-1680.

TREYBAL, R.E., 1981, Mass Transfer Operations, 3 ed., pp. 34 - 37, McGraw-Hill, Inc., Rohde Island.

WICKS, C.E. and WILSON, R.E., 1995, Fundamentals of Momentum, Heat, and Mass Transfer, 3 ed., pp. 493 - 498, 781 - 784, John Wiley \& Sons, Inc., New York.

\section{LIST OF SYMBOL}

a

$a_{A}, a_{B}, a_{C}$

$\mathrm{A}$

$\mathrm{A}_{0}$

$\mathrm{A}_{0 \mathrm{i}}$

$\mathrm{b}$

$\mathrm{b}_{\mathrm{A}}, \mathrm{b}_{\mathrm{B}}, \mathrm{b}_{\mathrm{C}}$

$\mathrm{B}$

$\mathrm{B}_{0}$

B0i

$\mathrm{C}_{\mathrm{A}}, \mathrm{C}_{\mathrm{B}}, \mathrm{C}_{\mathrm{C}}$

$\mathrm{C}_{\mathrm{A} 0}, \mathrm{C}_{\mathrm{B} 0}, \mathrm{C}_{\mathrm{C} 0}$

$\mathrm{C}_{\mathrm{U}}, \mathrm{C}_{\mathrm{H}}$

$\mathrm{C}_{\mathrm{UO}}, \mathrm{C}_{\mathrm{HO}}$

$\mathrm{C}_{\mathrm{U}^{*}}, \mathrm{C}_{\mathrm{H}^{*}} \quad:$ concentration of $\left(\mathrm{UO}_{2}{ }^{+2}\right)$ or $\left(\mathrm{MoO}_{2}^{+2}\right)$ and $\left(\mathrm{H}^{+}\right)$in source phase at membrane external interphase

$\mathrm{C}_{\mathrm{X}}, \mathrm{C}_{(\mathrm{RH}) 2}:$ concentration of $\left(\mathrm{UO}_{2} \mathrm{R}_{2} 2 \mathrm{HR}\right)$ or $\left(\mathrm{MoO}_{2} \mathrm{R}_{2} 2 \mathrm{HR}\right)$ and $(\mathrm{RH})_{2}$ in membrane phase

$\mathrm{C}_{\mathrm{X}^{*}}, \mathrm{C}_{(\mathrm{RH}) 2^{*}} \quad: \quad$ concentration of $\left(\mathrm{UO}_{2} \mathrm{R}_{2} 2 \mathrm{HR}\right)$ or $\left(\mathrm{MoO}_{2} \mathrm{R}_{2} 2 \mathrm{HR}\right)$ and $(\mathrm{RH})_{2}$ at membrane external interphase

$\mathrm{C}_{\mathrm{Xi}^{*}}, \mathrm{C}_{(\mathrm{RH}) 21^{*}}:$ concentration of $\left(\mathrm{UO}_{2} \mathrm{R}_{2} 2 \mathrm{HR}\right)$ or $\left(\mathrm{MoO}_{2} \mathrm{R}_{2} 2 \mathrm{HR}\right)$ and $(\mathrm{RH})_{2}$ at membrane internal interphase

$\mathrm{C}_{\mathrm{Ui}}, \mathrm{C}_{\mathrm{Hi}}:$ concentration of $\left(\mathrm{UO}_{2}^{+2}\right)$ or $\left(\mathrm{MoO}_{2}^{+2}\right)$ and $\left(\mathrm{H}^{+}\right)$in receiving phase 


\begin{tabular}{|c|c|}
\hline $\mathrm{C}_{\mathrm{Ui}^{*}}, \mathrm{C}_{\mathrm{Hi}^{*}}$ & $\begin{array}{l}: \text { receiving phase concentration of }\left(\mathrm{UO}_{2}^{+2}\right) \text { or }\left(\mathrm{MoO}_{2}^{+2}\right) \text { and }\left(\mathrm{H}^{+}\right) \\
\text {at membarane internal interphase }\end{array}$ \\
\hline Cuo & : initial concentration of $\left(\mathrm{UO}_{2}^{+2}\right)$ or $\left(\mathrm{MoO}_{2}^{+2}\right)$ in source phase \\
\hline $\mathrm{C}_{(\mathrm{RH}) 2 \mathrm{O}}$ & : initial concentration of $(\mathrm{RH})_{2}$ in membrane phase \\
\hline $\mathrm{C}_{\mathrm{HO}}$ & : initial concentration of $\left(\mathrm{H}^{+}\right)$in source phase \\
\hline $\mathrm{C}_{\mathrm{HOi}}$ & : initial concentration of $\left(\mathrm{H}^{+}\right)$in receiving phase \\
\hline $\mathrm{D}_{32}$ & : Sauter mean diameter \\
\hline $\mathrm{D}_{\mathrm{A}}, \mathrm{D}_{\mathrm{B}}, \mathrm{D}_{\mathrm{C}}$ & : diffusion coefficient of $\mathrm{A}, \mathrm{B}, \mathrm{C}$ in membrane phase \\
\hline $\mathrm{D}_{\mathrm{X}}, \mathrm{D}_{(\mathrm{RH}) 2}$ & $\begin{array}{l}\text { diffusion coefficient of }\left(\mathrm{UO}_{2} \mathrm{R}_{2} 2 \mathrm{HR}\right) \text { or }\left(\mathrm{MoO}_{2} \mathrm{R}_{2} 2 \mathrm{HR}\right) \text { and } \\
(\mathrm{RH})_{2} \text { in membrane phase }\end{array}$ \\
\hline $\mathrm{D}_{\mathrm{eX}}, \mathrm{D}_{\mathrm{e}(\mathrm{RH}) 2}$ & $\begin{array}{l}\text { effective diffusivity of }\left(\mathrm{UO}_{2} \mathrm{R}_{2} 2 \mathrm{HR}\right) \text { or }\left(\mathrm{MoO}_{2} \mathrm{R}_{2} 2 \mathrm{HR}\right) \text { and } \\
(\mathrm{RH})_{2} \text { in emulsion phase }\end{array}$ \\
\hline$D_{e A}, D_{e B}, D_{e C}$ & : effective diffusivity of $\mathrm{A}, \mathrm{B}, \mathrm{C}$ in emulsion phase \\
\hline $\mathrm{D}_{\mathrm{m}}$ & : diffusivity of solute in membrane phase \\
\hline $\mathrm{d}_{\mathrm{g}}$ & : average diameter of internal phase droplets \\
\hline $\mathrm{d}_{\mathrm{i}}$ & : drop diameter \\
\hline$d_{I}$ & $:$ is the impeller diameter \\
\hline$d_{32}$ & $:$ the Sauter mean diameter \\
\hline $\mathrm{g}$ & : acceleration of gravity \\
\hline $\mathrm{i}$ & : a sampling size interval \\
\hline $\mathrm{k}_{\mathrm{i}}$ & : membrane internal interphase mass transfer coefficient \\
\hline $\mathrm{k}_{\mathrm{o}}$ & : membrane external interphase mass transfer coefficient \\
\hline $\mathrm{K}_{\mathrm{i}}$ & : membrane internal resistance \\
\hline $\mathrm{K}_{\mathrm{o}}$ & : membrane external resistance \\
\hline $\mathrm{m}_{\mathrm{A}}, \mathrm{m}_{\mathrm{B}}, \mathrm{m}_{\mathrm{C}}$ & : distribution coefficient of $\mathrm{A}, \mathrm{B}, \mathrm{C}$ \\
\hline $\mathrm{M}_{\mathrm{B}}$ & : molecular weight of solvent B \\
\hline $\mathrm{M}_{\mathrm{R}}$ & : total number of moles per unit volume of raffinate phase \\
\hline $\mathrm{M}_{\mathrm{m}}$ & : total number of moles per unit volume of membrane phase \\
\hline $\mathrm{M}_{\mathrm{t}}$ & : total number of moles of extract phase \\
\hline $\mathrm{m}$ and $\mathrm{q}$ & : equilibrium constant \\
\hline $\mathrm{n}$ & : agitation speed \\
\hline ni & : number of drops in that interval \\
\hline $\mathrm{N}$ & : total number of globules in a batch \\
\hline $\mathrm{r}$ & : radial distance from globule center \\
\hline $\mathrm{R}$ & : Sauter mean radius of globules \\
\hline $\operatorname{Re}$ & : Reynolds number \\
\hline Sc & : Schmidt number \\
\hline Sh & : Sherwood number \\
\hline SMD & : Sauter mean diameter \\
\hline $\mathrm{t}$ & : time \\
\hline $\mathrm{T}$ & : temperature \\
\hline
\end{tabular}


$\mathrm{U}_{\mathrm{T}}, \mathrm{U}_{\mathrm{TS}} \quad$ : terminal velocity

$\mathrm{V}_{\mathrm{A}}, \mathrm{V}_{\mathrm{B}}, \mathrm{V}_{\mathrm{C}}$ : molar volume of $\mathrm{A}, \mathrm{B}, \mathrm{C}$

$\mathrm{V}_{\mathrm{e}} \quad: \quad$ volume of external phase

$\mathrm{V}_{\mathrm{i}} \quad$ : volume of internal phase

$\mathrm{V}_{\mathrm{m}} \quad$ : volume of membrane phase

$\mathrm{V}_{\mathrm{t}} \quad$ : total volume of a batch

$\mathrm{V}_{\mathrm{EO}} \quad:$ initial volume of emulsion phase

We : Weber number (dimensionless group)

$\mathrm{X}_{\mathrm{A}}, \mathrm{X}_{\mathrm{B}}, \mathrm{X}_{\mathrm{C}}:$ mole fraction of $\mathrm{A}, \mathrm{B}, \mathrm{C}$ in raffinate phase

$\mathrm{X}_{\mathrm{A} 0}, \mathrm{X}_{\mathrm{B} 0}, \mathrm{X}_{\mathrm{C} 0}$ : mole fraction of $\mathrm{A}, \mathrm{B}, \mathrm{C}$ at time $\mathrm{t} 0$

$\mathrm{y} \quad:$ dimensionless radial distance

$\mathrm{V}_{\mathrm{s}} \quad:$ slip velocity of dispersed drops to continous phase $(\mathrm{m} / \mathrm{s})$

VA : molar volume of solute A

\section{Greek letters}

$\begin{array}{ll}\varepsilon & : \text { volume fraction of internal phase in emulsion phase } \\ \phi & : \text { volume fraction of emulsion phase in a batch } \\ \tau & : \text { dimensionless time } \\ \alpha & : \text { partition coefficient of molybdenum } \\ \xi & : \text { association factor } \\ \rho & : \text { density of the external phase } \\ \rho \mathrm{e} & : \text { density of the fluid } \\ \eta & : \text { emulsion phase viscosity } \\ \mu & : \text { viscosity of solvent } \\ \mu \mathrm{e} & : \text { viscosity of the fluid } \\ \gamma & : \text { interfacial tension }\end{array}$


